\title{
Electrospun Polyaniline-Based Composite Nanofibers: Tuning the Electrical Conductivity by Tailoring the Structure of Thiol-Protected Metal Nanoparticles
}

\author{
Filippo Pierini, ${ }^{1}$ Massimiliano Lanzi, ${ }^{2}$ Paweł Nakielski, ${ }^{1}$ \\ and Tomasz Aleksander Kowalewski ${ }^{1}$ \\ ${ }^{1}$ Department of Biosystems and Soft Matter, Institute of Fundamental Technological Research, Polish Academy of Sciences, \\ ul. Pawinskiego 5B, 02-106 Warsaw, Poland \\ ${ }^{2}$ Department of Industrial Chemistry "Toso Montanari”, Alma Mater Studiorum, University of Bologna, Viale Risorgimento 4, \\ 40136 Bologna, Italy \\ Correspondence should be addressed to Filippo Pierini; fpierini@ippt.pan.pl
}

Received 30 March 2017; Revised 1 August 2017; Accepted 13 August 2017; Published 17 September 2017

Academic Editor: Mohammad Arjmand

Copyright (C) 2017 Filippo Pierini et al. This is an open access article distributed under the Creative Commons Attribution License, which permits unrestricted use, distribution, and reproduction in any medium, provided the original work is properly cited.

Composite nanofibers made of a polyaniline-based polymer blend and different thiol-capped metal nanoparticles were prepared using ex situ synthesis and electrospinning technique. The effects of the nanoparticle composition and chemical structure on the electrical properties of the nanocomposites were investigated. This study confirmed that Brust's procedure is an effective method for the synthesis of sub-10 nm silver, gold, and silver-gold alloy nanoparticles protected with different types of thiols. Electron microscopy results demonstrated that electrospinning is a valuable technique for the production of composite nanofibers with similar morphology and revealed that nanofillers are well-dispersed into the polymer matrix. X-ray diffraction tests proved the lack of a significant influence of the nanoparticle chemical structure on the polyaniline chain arrangement. However, the introduction of conductive nanofillers in the polymer matrix influences the charge transport noticeably improving electrical conductivity. The enhancement of electrical properties is mediated by the nanoparticle capping layer structure. The metal nanoparticle core composition is a key parameter, which exerted a significant influence on the conductivity of the nanocomposites. These results prove that the proposed method can be used to tune the electrical properties of nanocomposites.

\section{Introduction}

Organic electronics are based on the application of $\pi$ conjugated polymers (e.g., polyaniline and polythiophene), because they combine their excellent electrical and optical properties, with typical synthetic polymer properties such as flexibility, low cost, and good processability $[1,2]$. Onedimensional (1D) nanostructures have been studied in-depth in the field of organic electronics because of their small size and considerable elongation, which enable electrical carriers to move effectively along a controlled direction and make them suitable for the production of high-performance electronic systems with nanoscale dimension [3].
Electrospinning is the most attractive technique for the fabrication of continuous polymer $1 \mathrm{D}$ nanostructures with controllable diameter and compositions $[4,5]$. Furthermore, electrospinning leads to the fabrication of nanofibers with a favorable hierarchical structure for the development of advanced electronic devices, thanks to the stretching and alignment of polymer chains along the fiber axis [6], creating a preferential and more effective path for the charge transport into the material [7]. Unfortunately, intrinsically conductive polymer (ICP) solutions have an insufficient viscosity for the successful formation of nanofibers during the electrospinning process. However, electrospun ICP nanofibers can be easily fabricated by adding an auxiliary polymer (e.g., 
polyethylene oxide, $\mathrm{PEO}$ ) capable of increasing solution viscosity [8].

To improve and extend the electrical properties of polymer nanostructures, additional components are often incorporated to form nanocomposites [9]. Besides the formation of 1D nanostructured material, the addition of a secondary component to the conductive polymer blend to produce a nanocomposite is the most useful approach to improve and extend the functionality of conductive polymers [10]. Moreover, by combining nanofillers with 1D conductive polymers, new properties can be developed. For instance, original nanocomposites showing new optical, catalytic, and antibacterial properties can be obtained by incorporating metal nanoparticles into a polymer matrix [11-13].

Metal nanoparticles have attracted great interest due to their ease of synthesis and also because they are widely used as nanofillers for the development of conductive polymer composites (CPCs) [14]. Metal nanoparticles can be synthesized in situ in the polymer matrix [15]. In this synthetic route, nanoparticles grow inside the polymer matrix without agglomeration. In spite of this, with the in situ synthesis, it is not possible to finely control the spatial distribution of the fillers in the polymer matrix and it is also very difficult to remove the unreacted reagents from the polymer structure, negatively influencing the electrical properties of the final composites [16].

CPCs can be fabricated with an ex situ synthesis of metal nanoparticles and a subsequent introduction of the nanomaterials into the polymer matrix [17]. This procedure is particularly useful for the fabrication of conductive composites because it makes it possible to control filler distribution and to avoid the presence of undesirable components, which are key factors for developing effective nanostructures with enhanced electrical properties [16]. The most challenging aspect of this method is to successfully prepare stable nanoparticle colloidal systems that no longer have the tendency to aggregate and are incorporated more readily in the polymer [18]. In order to avoid these problems, we explored the possibility of using organic compounds (e.g., thiols, amines, and isocyanates), which have the ability to covalently interact with the surface of the metal nanoparticles in order to protect and stabilize the particle. The final nanoparticles have a metal core and organic shell structure where the conductive nucleus is surrounded by a chemically attached protective shell, which gives new properties to the materials [19].

Brust et al. developed a simple biphasic procedure to synthesize capped metal nanoparticles in organic solvents [20]. This technique enables the synthesis of nanoparticles made by different kinds of metal (e.g., gold, silver, and copper) as well as their alloys and to functionalize the particle surfaces with several types of molecules [21]. Since the thiol monolayer shell has dielectric properties, the choice of the capping agent is a crucial factor because it can affect the electrical properties of the core-shell nanoparticles and in turn the electrical conductivity of the final nanocomposites [22].

In this instance, we prepared electrospun polyaniline/polyethylene oxide/metal nanoparticle nanofibers using the ex situ technique. The electrospun nanostructured conductive composites were prepared by adding presynthesized thiol-capped metal nanoparticles made with different metal cores and protective shells in the polymer blend solution.

Ex situ synthesis of nanoparticles and the electrospinning technique have been investigated to fabricate nanocomposites with enhanced electrical conductivity. The chemical structure of metal nanoparticles was analyzed by attenuated total reflectance Fourier transform infrared (ATRFTIR), ultraviolet-visible spectroscopy (UV-Vis), and thermogravimetric analysis (TGA). The morphology of the prepared nanomaterials was characterized by transmission electron microscopy (TEM) and scanning electron microscopy (SEM). Finally, the structure of the electrospun materials was investigated by X-ray diffraction (XRD), and their electrical conductivity was evaluated by a four-point method. In addition, the structure of the nanofiller capping agents has been extensively studied since no in-depth data about its effect on the nanocomposite electrical properties have been found in the scientific literature. Our results confirm that the presence of capped metal nanoparticles in the polymer matrix of polyaniline-based nanofibers modifies the electrical conductivity of the final materials. Moreover, our study proves that it is possible to tailor nanocomposite properties by modifying the chemical structure and surface properties of the metal nanoparticles without changing the polymer blend composition and the nanofiller concentration.

\section{Materials and Methods}

2.1. Materials and Reagents. Polyaniline emeraldine base (molecular weight of $65000 \mathrm{~g} / \mathrm{mol}),( \pm)$-10-camphorsulfonic acid (HCSA), polyethylene oxide (PEO, molecular weight of $900000 \mathrm{~g} / \mathrm{mol})$, silver nitrate $\left(\mathrm{AgNO}_{3}\right)$, chloroauric acid $\left(\mathrm{HAuCl}_{4}\right), \quad 4$-methylbenzenethiol (MBT), 1-butanethiol $(\mathrm{BuT})$, potassium bromide $(\mathrm{KBr})$, tetraoctylammonium bromide (TOABr), sodium sulfate anhydrous $\left(\mathrm{Na}_{2} \mathrm{SO}_{4}\right)$, sodium borohydride $\left(\mathrm{NaBH}_{4}\right)$, chloroform $\left(\mathrm{CHCl}_{3}\right)$, toluene, dichloromethane $\left(\mathrm{CH}_{2} \mathrm{Cl}_{2}\right)$, and methanol $(\mathrm{MeOH})$ were purchased from Sigma-Aldrich. All chemicals were of analytical grade and were used as received without any further purification. Ultrapure water (resistivity: $18.5 \mathrm{M} \Omega \mathrm{cm}^{-1}$ ) used for the experiments and all cleaning steps was obtained from a Milli-Q Advantage A10 Water Purification System.

2.2. Electrospun Nanomaterial Fabrication. Colloidal metal protected nanoparticles were synthesized according to Brust's synthesis protocol (see Supplementary Material and Table S1 available online at https://doi.org/10.1155/2017/6142140 for nanoparticle synthesis details).

The starting polymer blend and polymer composite solutions were prepared according to an already standardized method [7]. The concentration of nanoparticles in the polymer solutions was previously optimized for the production of nanocomposites with the largest possible amount of nanofillers, which does not cause any nanofiber structural defect.

Polyaniline $(500 \mathrm{mg})$ and HSCA $(645 \mathrm{mg})$ were dissolved in $50 \mathrm{ml}$ of $\mathrm{CHCl}_{3}$ and stirred for $48 \mathrm{~h} .500 \mathrm{mg}$ of PEO was 


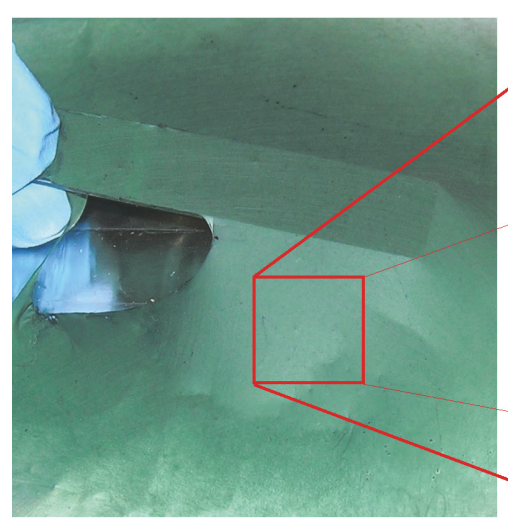

(a)

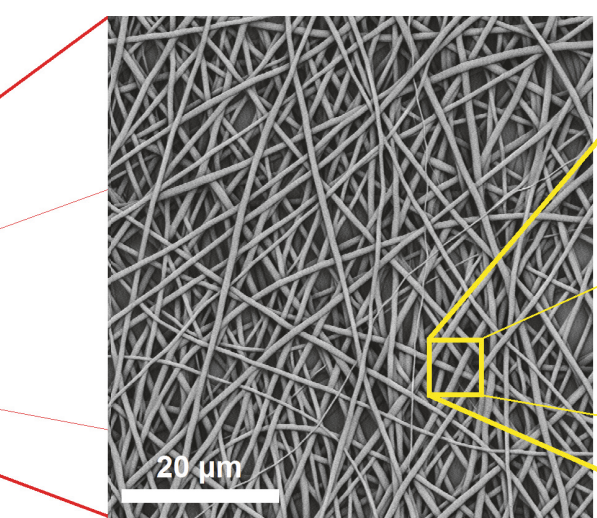

(b)

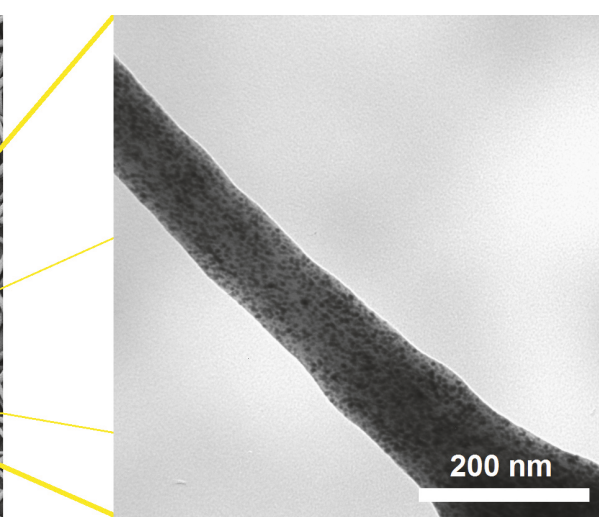

(c)

FIGURE 1: Hierarchical structure of the composite nanofiber mats: (a) photograph of an electrospun nanocomposite; (b) SEM image of an electrospun mat made by randomly oriented conducting polymer/nanoparticle composite nanofibers; (c) TEM micrograph of an electrospun nanofiber containing thiol-capped metal nanoparticles.

TABLE 1: Electrospinning parameters applied in the fibrous mat fabrication.

\begin{tabular}{lccc}
\hline Sample & $\begin{array}{c}\text { Solution flow rate } \\
(\mu \mathrm{l} / \mathrm{min})\end{array}$ & $\begin{array}{c}\text { Applied voltage } \\
(\mathrm{kV})\end{array}$ & $\begin{array}{c}\text { Needle-target distance } \\
(\mathrm{cm})\end{array}$ \\
\hline Neat nanofibers & 5.0 & 9.0 & 15.0 \\
PANI/Au-MBT & 5.0 & 8.0 & 12.0 \\
PANI/Au $\mathrm{Ag}_{1}-\mathrm{MBT}$ & 5.0 & 7.0 & 10.0 \\
PANI/Au $\mathrm{Ag}_{4}-\mathrm{MBT}$ & 5.0 & 7.0 & 10.0 \\
PANI/Ag-MBT & 5.0 & 8.0 & 8.0 \\
PANI/Ag-BuT & 5.0 & 8.0 & 8.0 \\
\hline
\end{tabular}

added to the solution and stirred magnetically for about $24 \mathrm{~h}$. This solution was filtered using a $0.45 \mu \mathrm{m}$ PTFE filter to obtain a homogeneous solution. The polymer blend solution was electrospun to produce a reference material and it was also used as a starting solution to prepare the composite samples.

We prepared polyaniline/polyethylene oxide/metal nanoparticle nanofibers with well-defined and controlled hierarchical structure using ex situ technique and electrospinning (Figure 1). The composite solutions were prepared by adding $5.0 \mathrm{mg}$ of nanoparticles (7.06 wt\% of the final dry composite) to $2.0 \mathrm{ml}$ of polymer blend solution. The suspensions were stirred for $30 \mathrm{~min}$ and repeatedly sonicated for a few seconds. Following this, all the solutions were immediately electrospun.

The processed polymer solutions were loaded in a $2 \mathrm{ml}$ syringe connected to a metal needle. A syringe pump (KD Scientific syringe pump model 200-CE) provided a constant and controlled solution flow rate, while a high-voltage power supply (Spellman model SL150) was employed to apply the potential needed to perform the electrospinning. Lastly, a glass slide was placed over a metal collector, which was horizontally placed a few centimeters from the needle in order to collect the nanofibers. Suspensions were electrospun according to the experimental parameters reported in Table 1.

\subsection{Material Characterizations}

2.3.1. Investigation of Nanomaterial Morphology. The morphology and dimensions of the metal nanoparticles as well as the presence and distribution of nanofillers within the electrospun nanofibers were assessed by a transmission electron microscope (TEM, Philips, CM100) operating at an accelerating voltage of $100 \mathrm{kV}$ for the inorganic materials and $80 \mathrm{kV}$ for the composites. The particle size was determined manually by measuring the diameter of 100 nanoparticles. It is worth mentioning that the visualized electrospun nanofiber size is affected by the polymer-beam interaction, which causes the nanofibers to stretch. Therefore, TEM images were not used to evaluate the nanocomposite nanofiber diameter.

Nanofiber morphology was characterized using a scanning electron microscope (SEM, Carl Zeiss, EVO MA 10) at $12 \mathrm{kV}$ acceleration voltage and $8 \mathrm{~mm}$ working distance. The analyzed polymer samples were sputter-coated with a layer of gold prior to the observation. The mean nanofiber diameter was evaluated analyzing more than 100 nanofibers from each of the samples.

2.3.2. Thermogravimetric Analysis (TGA). The amount of capping agent covalently bonded onto the metal nanoparticle surface was evaluated by thermogravimetric analyses (TGA, TA Instruments, SDT Q600). The analysis was carried out 
testing $5.0 \mathrm{mg}$ of nanoparticles in the temperature range from $25^{\circ} \mathrm{C}$ to $600^{\circ} \mathrm{C}$ at a rate of $10^{\circ} \mathrm{C} / \mathrm{min}$ and under a nitrogen flow of $100 \mathrm{ml} / \mathrm{min}$.

2.3.3. Spectroscopic Characterizations. Attenuated total reflectance Fourier transform infrared (ATR-FTIR) spectra of nanoparticles were recorded using a Nicolet 380 FTIR spectrometer equipped with a germanium crystal in order to study the chemical composition of the nanoparticle surface. The spectra were collected between 500 and $4000 \mathrm{~cm}^{-1}$ at a resolution of $2 \mathrm{~cm}^{-1}$ after depositing $5.0 \mathrm{mg}$ of nanoparticles on the ATR crystal.

UV-visible absorption measurements (UV-Vis) of nanoparticle dispersions were measured using a Varian Cary 300 Bio UV-Vis Spectrophotometer. The spectra were collected from 350 to $650 \mathrm{~nm}$ at a resolution of $1 \mathrm{~nm}$ in order to evaluate the surface plasmon resonance (SPR) of metal nanoparticles.

$\mathrm{X}$-ray diffraction (XRD) analyses of the polymer materials were performed using a Philips PW1050/81-PW1710 diffractometer using $\mathrm{Cu} \mathrm{K} \alpha$ radiation.

\subsubsection{Electrical Conductivity Measurement. The electrical} conductivity of the electrospun mats was investigated by our routine method based on a four-point probe system $[7,8]$. The analyzed samples were prepared by collecting around $50 \mathrm{mg}$ of polymer blend/composite nanofibers on the glass slides placed onto the metal collector during the electrospinning process. The cross-sectional thickness (from $100 \mu \mathrm{m}$ to $150 \mu \mathrm{m}$ ) and homogeneity of the inner structure of the sample were evaluated by SEM microscopy prior to the electrical characterization. The four probes of an Alessi Instruments C4S four-point probe head (probe material: osmium; probe tip spacing: $1.6 \mathrm{~mm}$; spring pressure: $40-70 \mathrm{~g}$; probe tip diameter: $0.2 \mathrm{~mm}$ ) were lowered onto the sample. In order to avoid any problem related to the probe-sample connection, the probe surface was covered with a small amount of silver paint by microdeposition technique. Data were acquired using a Keithley 2401 SourceMeter at room temperature and the results were calculated averaging the data obtained measuring the conductivity in 10 different positions on three different mats per each sample.

\section{Results and Discussion}

Before studying the composite materials, capped metal nanoparticles used as nanofillers were deeply investigated. Figure 2 shows TEM images of all synthesized nanoparticles. As it is visible, metal nanoparticles show spherical morphology of almost identical diameter. Furthermore, the nanoparticles do not tend to aggregate because of the thiol layer stabilization. TEM micrographs revealed particles with size lower than $10 \mathrm{~nm}$ with narrow distribution. The average nanoparticle diameter is around $5 \mathrm{~nm}$. The composition and capping agent do not influence the morphology and dimension of the nanoparticle except for $\mathrm{Au}_{1} \mathrm{Ag}_{4}$-MBT nanoparticles which present the same morphology and diameter but a slightly broader size distribution. Ristig et al. have recently demonstrated that capped AuAg alloy nanoparticles have a metal core-shell structure with an Au-rich core and an Agrich shell at a low silver content [23]. When the amount of silver is predominant, the distribution of metals into the nanoalloys structure is stochastic and a single particle contains more than one crystallite. The different size distribution of $\mathrm{Au}_{1} \mathrm{Ag}_{4}$-MBT results from a change in the nucleation and growth mechanism if compared with the other nanoparticles.

The composition and chemical structure of nanoparticles were studied to investigate their influence on the final composite electrical properties. Capped metal nanoparticles show surface plasmon resonance (SPR), due to the interaction between light and metal electrons in the conduction band which collectively oscillate in resonance at specific wavelength. SPR wavelength varies with particle size, shape, and metal type $[24,25]$. The continuous change in the SPR absorption maximum as a function of the $\mathrm{Au}$ : $\mathrm{Ag}$ molar ratio can be used to accurately quantify the composition of alloy nanoparticles [23]. Our UV-Vis results (Figure 3(a)) revealed that Ag-MBT nanoparticles show a SPR peak at $395 \mathrm{~nm}$, while the Au-MBT nanoparticles absorption maximum is centred at $530 \mathrm{~nm}$. We noticed that as the Au molar ratio increases, the SPR maximum is linearly red-shifted. These results (Figure 3(b)) confirm the synthesis of nanoparticles with different metal and the possibility of quantitatively controlling the $\mathrm{Au}$ : Ag molar ratio.

At this step, we focused on the characterization of silver nanoparticle surface protected with different thiol molecules. ATR-FTIR spectra of Ag-BuT and Ag-MBT nanoparticles (Figure 4) revealed the presence of the specific absorption peaks related to 1-butanethiolate and 4methylbenzenethiolate groups, respectively (Table S2) [20, $26,27]$. It is interesting to notice that the methyl and methylene signals of Ag-BuT result slightly shifted when compared with the same absorption band registered for crystalline long chain alkanes. This difference suggests a low degree of order in the butyl chains attached onto the nanoparticles, which are free to move and do not form well-defined supramolecular structures [28]. Moreover, it is also important to highlight the absence of the $\mathrm{S}-\mathrm{H}$ absorption peak at around $2580 \mathrm{~cm}^{-1}$ in both spectra, which proves the involvement of the sulfur atoms in the chemisorption of thiol molecules on the metal nanoparticles surface [27].

Figure 5 shows typical TGA results of Ag-BuT and AgMBT nanoparticles. TGA is a valid method for quantitative determination of the organic molecules attached onto the nanoparticle surface [29]. TGA curve of Ag-BuT shows that the mass of the sample initially slowly decreases but then the mass loss drastically increases before $200^{\circ} \mathrm{C}$. The total mass loss for this sample is almost $16 \%$ when heated to $600^{\circ} \mathrm{C}$. In the case of $\mathrm{Ag}-\mathrm{MBT}$, the total mass loss is more than $20 \%$, and sample decomposition begun at higher temperature when compared to the Ag-BuT sample. Moreover, the mass loss took place in a narrower range of temperature. The difference in mass loss between the analyzed nanoparticles is consistent with the molecular mass of the capping agents. Since the molecular weight of MBT is higher than this of $\mathrm{BuT}$, the analysis confirms that the number of thiol molecules attached onto the metal surface of Ag-BuT and Ag-MBT 


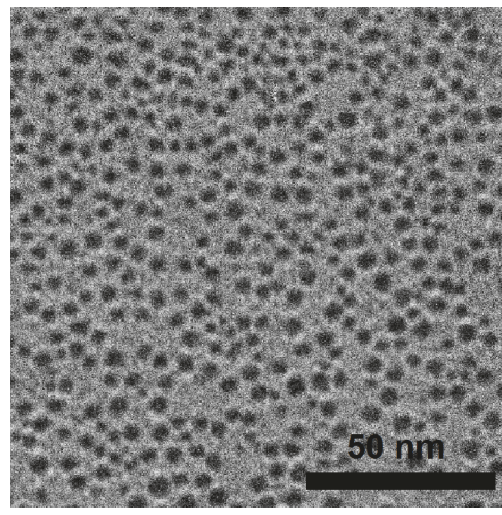

(a)

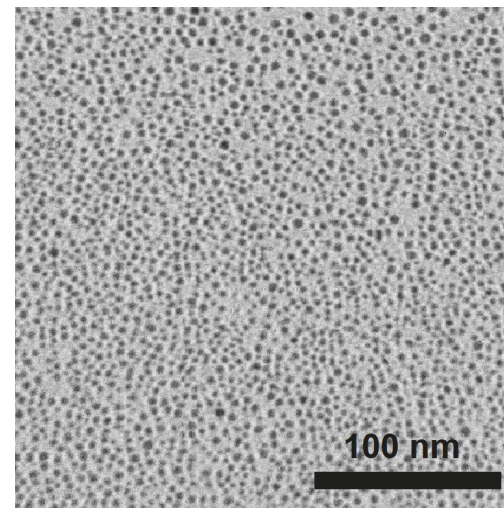

(b)

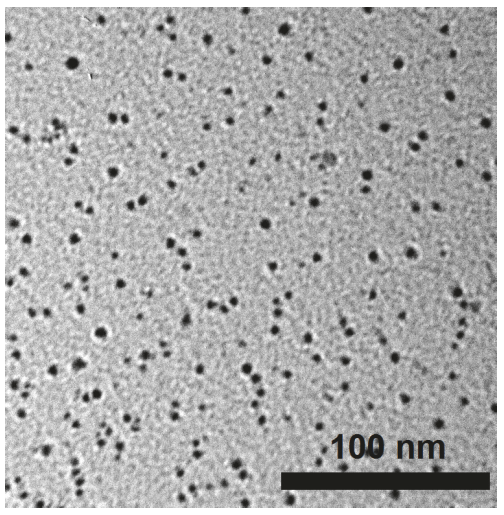

(c)

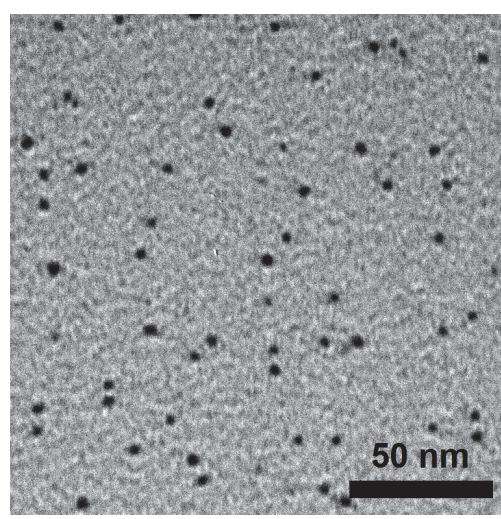

(d)

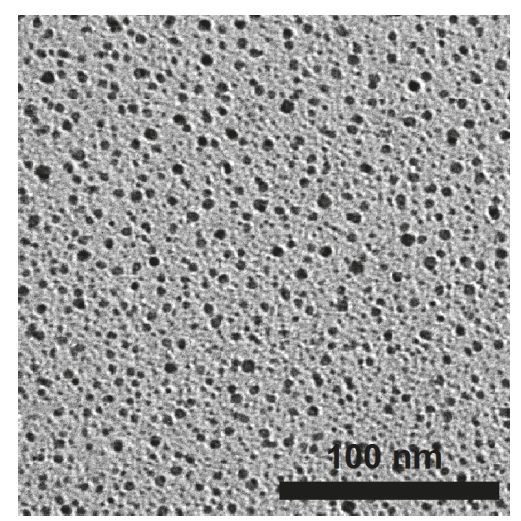

(e)

Figure 2: TEM images of metal nanoparticles capped with different thiols: (a) Ag-BuT; (b) Ag-MBT; (c) Au-MBT; (d) Au $\mathrm{Ag}_{1}-\mathrm{MBT}$; (e) $\mathrm{Au}_{1} \mathrm{Ag}_{4}$-MBT.

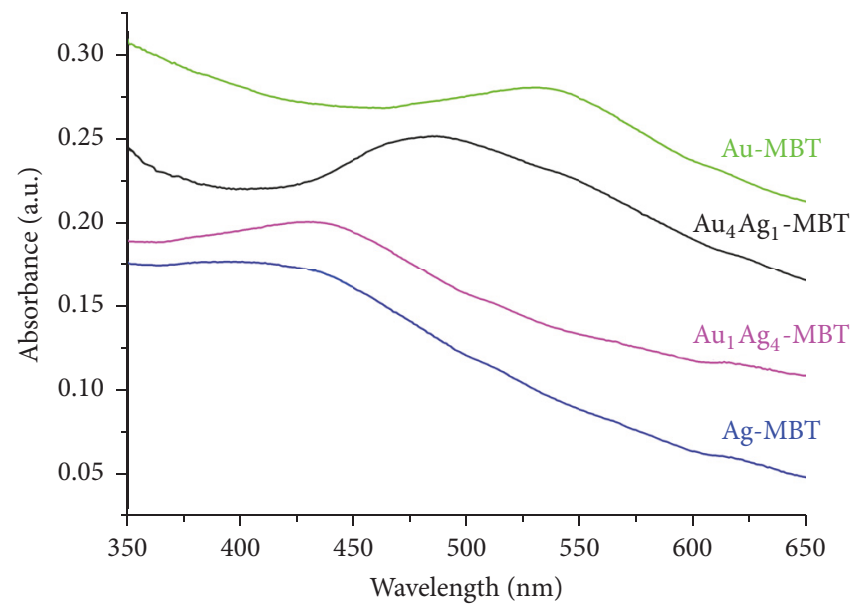

(a)

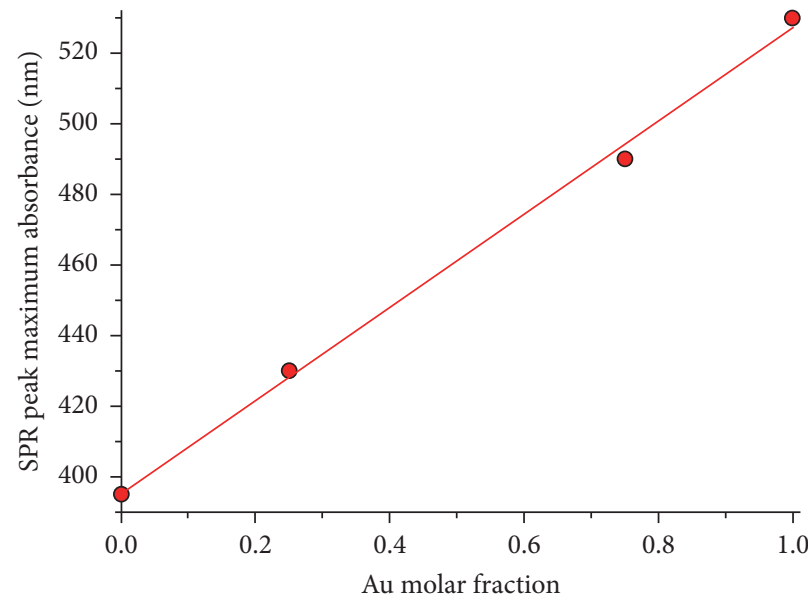

(b)

FIGURE 3: Influence of metal core composition on the UV-Vis absorption spectrum of nanoparticles capped with MTB: (a) surface plasmon resonance absorption spectra of metal protected nanoparticle at different $\mathrm{Au}$ : Ag molar ratio; (b) nanoparticle gold molar fraction as a function of the maximum surface plasmon resonance peak. 


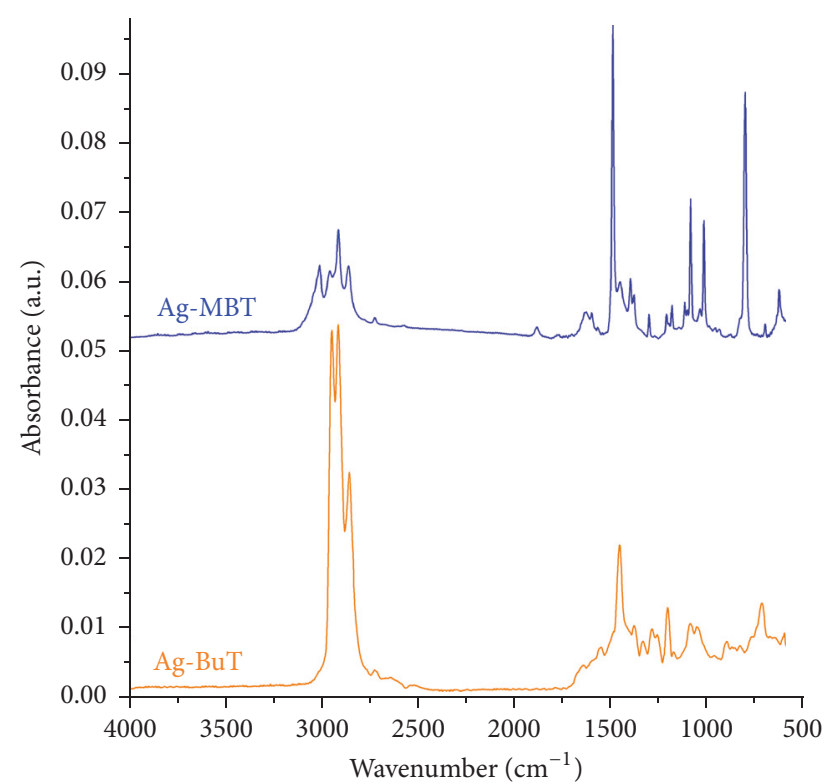

FIGURE 4: Chemical structure of the nanoparticle protective layers: comparison of ATR-FTIR spectra between Ag-BuT and Ag-MBT nanoparticles prepared as part of this study.

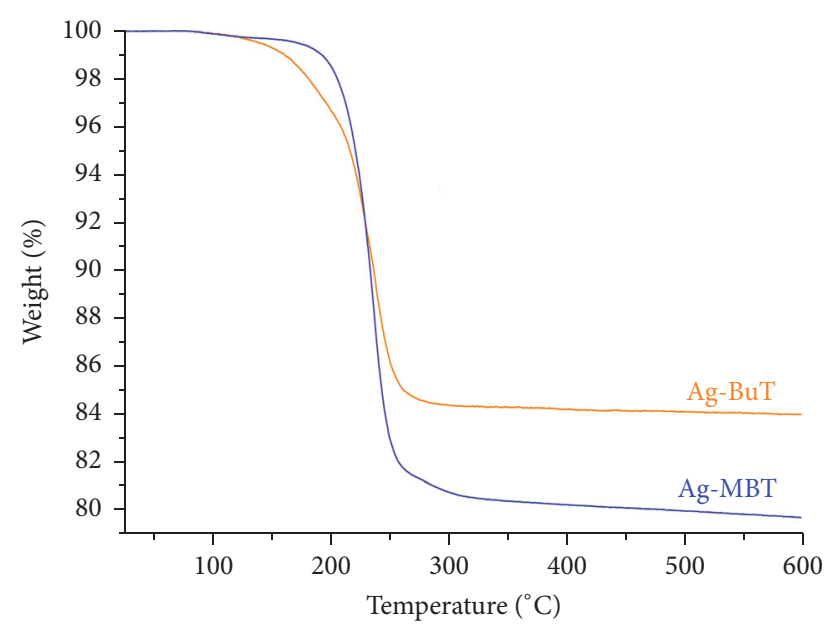

FIGURE 5: Quantification of nanoparticle coatings: mass percent versus temperature TGA curves of Ag-BuT and Ag-MBT nanoparticles.

nanoparticles is comparable. Ag-MBT nanoparticles show greater stability due to the higher boiling point of the ligand [30]. The Ag-BuT degradation took place in a wider range of temperatures which is an indicator of the degree of disorder in interchain interactions, especially when compared with $\mathrm{Ag}$ MBT nanoparticles where the $\pi-\pi$ stacking of aromatic rings can create stable supramolecular structures [31].

The TGA data confirm the previous ATR-FTIR, which proves the formation of different protective layers onto the nanoparticle surface with similar morphology. The protective layers are similar in molecular density but show different length and structuration.

To investigate the morphological properties of electrospun nanofibers, the prepared samples were analyzed by SEM.
Figure 6 shows SEM micrographs of neat electrospun fibers and a representative nanocomposite sample. The electrospun nanofibers show a continuous cylindrical shape without defects and a randomly oriented disposition. The materials had the typical electrospun mat structure where the nanofibers are in contact with each other forming a three-dimensional network. The blend fiber surfaces were quite smooth when compared with typical conjugated polymer/PEO nanofibers, which often show a textured surface [32]. Nanocomposite fiber morphology appeared to be unaffected by the presence of nanofillers, which do not induce the formation of beads or blobs into the mat structure. The presence of nanoparticles is not clearly visible in these images, since the majority of the particles are embedded into the fiber structure. Table 2 reports the diameter values of the electrospun fibers. The average diameter of neat fibers was $565 \mathrm{~nm}$ with a narrow distribution. The inclusion of metal capped nanoparticles does not cause a relevant variation in fiber diameter. This is due to the mutual effect of the polymer solution electrical conductivity and viscosity, as both of these quantities increase after the addition of nanofillers. In fact, the electrospun fiber diameter usually increases proportionally with the increment of solution viscosity [33]. On the other hand, this effect is counterbalanced by the increment in electrical conductivity of the starting solution, which promotes the formation of thinner nanofibers, due to the increment of charge density on the electrospinning jet which in turn promotes the jet elongation along its axis, reduces the Rayleigh instability, and enhances the whipping instability [34]. The composite nanofibers show a slightly broader diameter distribution. The fabrication of nanofibers with comparable diameters for each composition is a key factor for the successful completion of the experiment, as their electrical conductivity is influenced by the nanofiber size [35].

With TEM, we can determine the internal morphological structure of the composites on the nanometer scale. Figure 7 shows the TEM images of all the fabricated nanocomposites. The images reveal that metal nanoparticles were homogeneously distributed within the fibers and their spherical shape remained unchanged after the electrospinning process for all the fabricated nanocomposites. In the images, it can be observed that nanofillers are randomly embedded into the electrospun nanofibers. The nanoparticles are well-dispersed into the matrix and they do not exhibit any tendency to aggregate. Furthermore, the composite nanomaterials have a similar nanofillers distribution for each composition, demonstrating that the composite nanofiber inner morphological structure is not influenced by the nanoparticle chemical composition.

The chemical nanofibers structures were investigated by XRD. Since it is well known that the presence of metal nanofillers within the polymer matrix can change the material crystallinity (and, in turn, the final material electrical properties) [36], we focused on the influence of nanoparticles upon the polymer chain conformation. As shown in Figure 8, all the electrospun samples showed the typical peaks associated with the electrospun polymer blend in their XRD patterns [7]. In XRD diffractograms, two typical peaks at $2 \theta=19.1^{\circ}$ and $23.3^{\circ}$, which correspond to semicrystalline PEO, can 


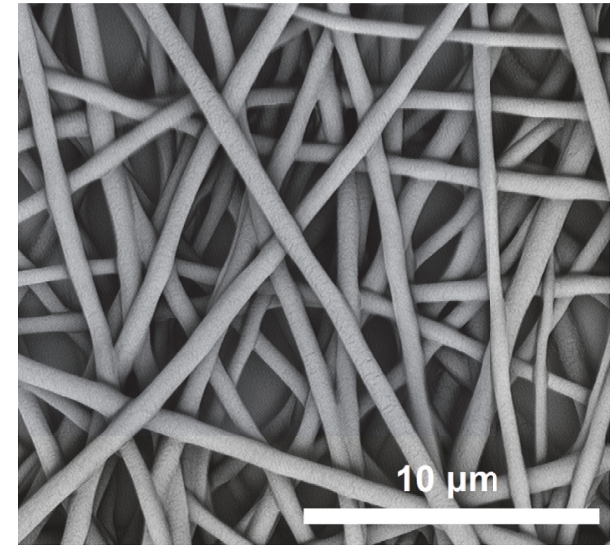

(a)

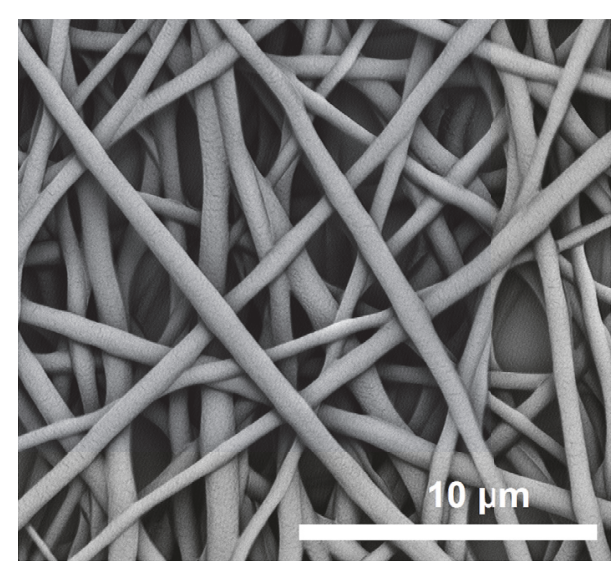

(b)

FIGURE 6: Effect of nanofillers on the morphological properties of electrospun mats: SEM images of electrospun (a) neat polyaniline-based and (b) PANI/Ag-MBT nanofibers.

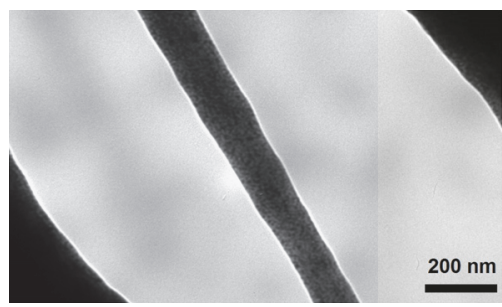

(a)

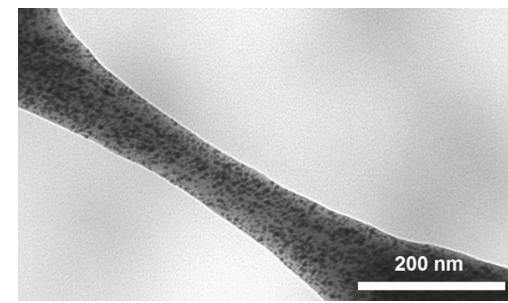

(b)

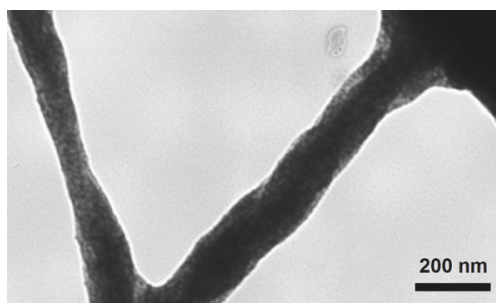

(c)

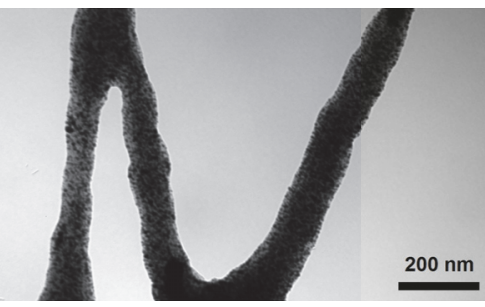

(d)

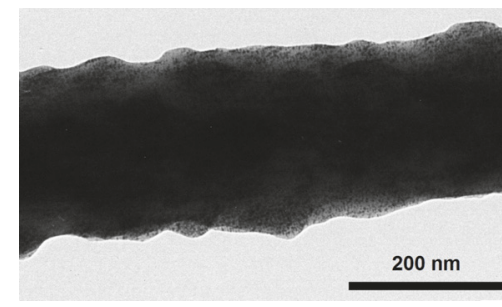

(e)

Figure 7: Representative TEM micrographs of electrospun composite nanofibers: (a) PANI/Ag-BuT; (b) PANI/Ag-MBT; (c) PANI/Au$\mathrm{MBT}$; (d) PANI/Au $\mathrm{Ag}_{1}-\mathrm{MBT}$; (e) PANI/ $\mathrm{Au}_{1} \mathrm{Ag}_{4}-\mathrm{MBT}$. The black dots are metal nanoparticles uniformly embedded within the electrospun nanofibers.

TABLE 2: Diameter of nanofibers and electrical conductivity of the electrospun nanomaterials. Data are reported as mean \pm standard deviation.

\begin{tabular}{lcc}
\hline Sample & Fiber diameter $(\mathrm{nm})$ & Electrical conductivity $($ S/cm $)$ \\
\hline PANI & $565 \pm 75$ & $6.81 \times 10^{-8} \pm 3.8 \times 10^{-9}$ \\
PANI/Au-MBT & $635 \pm 135$ & $5.20 \times 10^{-7} \pm 3.6 \times 10^{-8}$ \\
PANI/Au ${ }_{4}$ Ag $_{1}$-MBT & $690 \pm 116$ & $2.61 \times 10^{-7} \pm 3.4 \times 10^{-8}$ \\
PANI/Au Ag $_{4}$-MBT & $678 \pm 127$ & $3.16 \times 10^{-7} \pm 4.2 \times 10^{-8}$ \\
PANI/Ag-MBT & $648 \pm 109$ & $9.18 \times 10^{-7} \pm 8.5 \times 10^{-8}$ \\
PANI/Ag-BuT & $611 \pm 101$ & $3.19 \times 10^{-6} \pm 2.9 \times 10^{-7}$ \\
\hline
\end{tabular}




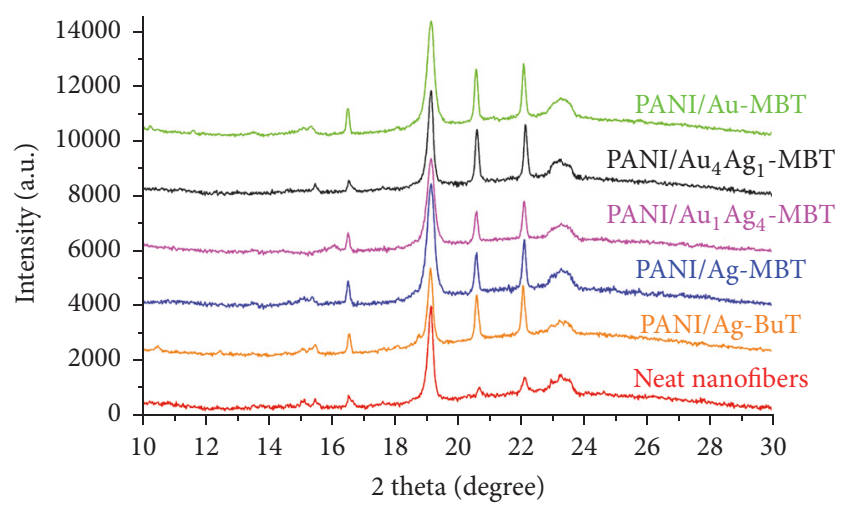

FIGURE 8: Molecular structure of the electrospun nanofiber polymer matrix: XRD pattern of neat polyaniline-based nanofibers together with the XRD diffractograms of the electrospun nanocomposites obtained by embedding several kinds of nanofillers into the polymer structure.

be clearly detected [8]. Furthermore, three weak peaks are visible in this region. The patterns show a very broad signal centred at around $2 \theta=23^{\circ}$, which is ascribable to the amorphous structure of polyaniline $[7,8]$. The neat polymer and composite mats showed similar patterns, without any additional peaks, which proves that the presence of embedded nanoparticles does not induce the formation of defects. Small differences in the peak intensities are noticeable; therefore, PEO peaks appear slightly sharper and of higher intensity in the composite nanofiber diffractograms. The presence of nanofillers causes only a small rise in PEO crystallinity and does not influence the polyaniline chain arrangement; hence its structure remains amorphous. Higher crystallinity content is usually observed in nanocomposites, which is due to the nucleation effect of nanoparticles dispersed into the polymer matrix [37]. The polymer crystallinity degree in the fabricated electrospun nanocomposites is not considerably higher because the rapid evaporation of the solvent during electrospinning prevents crystal nucleation [38]. The XRD diffractograms of the nanocomposites show similar patterns and signal intensity, which proves the presence of similar chemical structures and confirms the previous TEM analysis results.

In order to understand the impact of chemical and structural features of nanofillers on the electrical properties of electrospun nanomaterials, we studied the electrical conductivity $(\sigma)$ of the produced mats. The electrical properties of the electrospun mats were evaluated by a four-point method at $25^{\circ} \mathrm{C}$. The results of four-point tests are shown in Table 2 . The neat nanofibers showed very low conductivity $\left(\sigma=6.81 \times 10^{-8} \mathrm{~S} / \mathrm{cm}\right)$. The main reason for this feature is connected to the presence of PEO in the polymer blend, which undoubtedly helps the formation of nanofibers but at the same time drastically reduces electrical conductivity because of its distinctive dielectric properties.

The presence of nanoparticles causes noticeable increase of the electrical conductivity of polymer mats. Among the developed nanomaterial samples, the one with the best electrical properties showed a remarkable improvement in conductivity, which was increased by two orders of magnitude $\left(\sigma=3.19 \times 10^{-6} \mathrm{~S} / \mathrm{cm}\right)$, when Ag-BuT nanoparticles were used as nanofillers. The improved conductivity of PANI/Ag$\mathrm{BuT}$ nanocomposite arises from the dispersion of nanoparticles with high electrical conductivity in the polymer matrix which favors better charge transport. The addition of foreign materials (e.g., nanoparticles) into the ICP matrix may create a discontinuity in the charge carriers hopping motion [7]. As a result, electrical conductivity greatly differs according to the ability of the nanofillers to allow (or not) the passage of electrical charges. The greater the dielectric property of the discontinuity points added in the polymer structure is, the lower the value of electrical conductivity of the composite will be.

As shown in Table 2, the electrical conductivity of the PANI/Ag-MBT $\left(\sigma=9.18 \times 10^{-7} \mathrm{~S} / \mathrm{cm}\right)$ nanocomposite is higher than that of the neat fibers but is lower than that of PANI/Ag-BuT. All the metal particles we used were covered by a protective monolayer of organic molecules, which can be considered as a monomolecular insulating layer acting as a barrier for the charge transport. The structure of the protective layer strongly affects the metal nanoparticle electrical properties [22]. As a result, when these particles are used as nanofillers, the type, thickness, and conformation of the capping layer intensively influence the interfacial electronic interaction between polymer semiconductor chains and the metal core. Electrical conductivity of PANI/Ag-MBT is lower than PANI/Ag-BuT because, as it is proved, BuT chemical structure is smaller than that of MBT and its related capping layer is thinner.

The conductivity of the PANI/Au-MBT $(\sigma=5.20 \times$ $10^{-7} \mathrm{~S} / \mathrm{cm}$ ) is still larger than the neat polymer nanofibers, but it is about half as much as that of PANI/Ag-MBT. Since the nanofibers have the same hierarchical structure, the variation of electrical properties is due to the metal core type. This result is in accordance with the conductivity values for the metals we used in our experiment, as silver is a better conductor of electricity than gold.

Electrical properties of the nanocomposites made by gold-silver alloy nanoparticles (with $\mathrm{Au}: \mathrm{Ag}$ molar ratio of $1: 4$ and $4: 1$ ), protected with MBT, have values that are similar to each other. PANI $/ \mathrm{Au}_{1} \mathrm{Ag}_{4}-\mathrm{MBT}$ electrical conductivity is slightly greater than PANI/ $\mathrm{Au}_{4} \mathrm{Ag}_{1}-\mathrm{MBT}$ due to the higher amount of silver in the nanoparticle metal core. On the other hand, it is surprising that their electrical conductivity values are not included within the range of PANI/Au-MBT at one end and PANI/Ag-MBT at the other. Electrospun nanocomposites made by gold-silver alloy nanoparticles have lower conductivity even than PANI/Au-MBT, in spite of the presence of silver in their cores. Although the reason why this is the case is not completely clear, this behavior might be connected to the metal core structure. In particular, it may be due to the particle heterogeneity caused by the presence of different crystallites within the nanoparticle core. However, these results prove the fundamental role of the nanoparticle metal core composition. Therefore, the electrical properties of the developed electrospun nanocomposites are deteriorated 
by the presence of a second metal type in the nanoparticle structure.

\section{Conclusions}

Electrospun polyaniline-based composite nanofibers were successfully fabricated by ex situ synthesis of thiol-protected metal nanoparticles. Nanoparticles with different metal core compositions and two different capping agents were synthesized according to Brust's procedure and they were fully characterized to confirm their morphological, chemical, and structural properties before their inclusion in the polymer matrix.

SEM analysis of electrospun nanomaterials revealed that nanofibers have defect-free cylindrical morphology, with similar fiber diameter and orientation. TEM analysis showed uniform dispersion of the spherically shaped metal nanoparticles in all the electrospun polymer nanofibers. The XRD patterns indicated that the conjugated polymer chain arrangement is not affected by the presence of nanofillers and that the polymer matrix structure is not influenced by the metal nanoparticle core and thiol protecting layer composition.

Electrical conductivity measurements performed by a four-probe method revealed that the PANI/Ag-BuT nanocomposite exhibits remarkably improved electrical conductivity when compared with the electrospun neat polymer material. More interestingly, the PANI/Ag-BuT nanocomposite exhibits larger electrical conductivity than those of PANI/Ag-MBT, thus confirming the pivotal role of the thiol layer structure in order to produce polymer nanocomposites with desirable electrical properties. Four-probe test results confirmed that the electrical conductivity of the developed electrospun nanocomposites is also influenced by the metal core composition, as PANI/Ag-MBT showed better electrical properties than PANI/Au-MBT. Furthermore, it was found that the use of a gold-silver alloy nanoparticle protected with the same thiol as nanofillers causes a decline in nanocomposite electrical conductivity, proving the important role played by metal core nanoparticle uniformity of composition.

This study demonstrated that designing metal nanoparticles with well-defined chemical composition and capping molecule structure will pave the way for an effective strategy to fine-tune the electrical properties of conjugated polymerbased nanocomposites.

\section{Conflicts of Interest}

The authors declare that they have no conflicts of interest.

\section{Acknowledgments}

Filippo Pierini wishes to gratefully acknowledge the support of National Science Centre (NCN Grants nos. 2015/19/D/ ST8/03196 and 2011/03/B/ST8/05481). Experiments were performed using equipment made available thanks to EC structural funds (Project POIG no. 02.02.00-17-024/08-00). The authors would also like to acknowledge the University of Bologna (Selected Topics Research Fund) for supporting this research.

\section{References}

[1] F. Pierini, M. Lanzi, P. Nakielski et al., "Single-material organic solar cells based on electrospun fullerene-grafted polythiophene nanofibers," Macromolecules, vol. 50, no. 13, pp. 49724981, 2017.

[2] M. Lanzi, L. Paganin, F. Pierini, F. Errani, and F. P. DiNicola, "Use of poly(3-methylthio)thiophene blends for direct laser tracing and bulk heterojunction solar cells," Reactive and Functional Polymers, vol. 83, pp. 33-41, 2014.

[3] M. Zhi, A. Manivannan, F. Meng, and N. Wu, "Highly conductive electrospun carbon nanofiber/MnO 2 coaxial nano-cables for high energy and power density supercapacitors," Journal of Power Sources, vol. 208, pp. 345-353, 2012.

[4] W. E. Teo and S. Ramakrishna, "A review on electrospinning design and nanofibre assemblies," Nanotechnology, vol. 17, no. 14, article R01, pp. R89-R106, 2006.

[5] X. Shi, W. Zhou, D. Ma et al., "Electrospinning of nanofibers and their applications for energy devices," Journal of Nanomaterials, vol. 2015, Article ID 140716, 20 pages, 2015.

[6] D. Kołbuk, P. Sajkiewicz, and T. A. Kowalewski, "Optical birefringence and molecular orientation of electrospun polycaprolactone fibers by polarizing-interference microscopy," European Polymer Journal, vol. 48, no. 2, pp. 275-283, 2012.

[7] F. Pierini, M. Lanzi, I. G. Lesci, and N. Roveri, "Comparison between inorganic geomimetic chrysotile and multiwalled carbon nanotubes in the preparation of one-dimensional conducting polymer nanocomposites," Fibers and Polymers, vol. 16, no. 2, pp. 426-433, 2015.

[8] F. Pierini, M. Lanzi, P. Nakielski, S. Pawłowska, K. Zembrzycki, and T. A. Kowalewski, "Electrospun poly(3-hexylthiophene)/ poly(ethylene oxide)/graphene oxide composite nanofibers: effects of graphene oxide reduction," Polymers for Advanced Technologies, vol. 27, no. 11, pp. 1465-1475, 2016.

[9] S. H. Lee, J. H. Kim, S. H. Choi, S. Y. Kim, K. W. Kim, and J. R. Youn, "Effects of filler geometry on internal structure and physical properties of polycarbonate composites prepared with various carbon fillers," Polymer International, vol. 58, no. 4, pp. 354-361, 2009.

[10] Z. Zhang, F. Zhang, X. Jiang, Y. Liu, Z. Guo, and J. Leng, "Electrospinning and microwave absorption of polyaniline/ polyacrylonitrile/multiwalled carbon nanotubes nanocomposite fibers," Fibers and Polymers, vol. 15, no. 11, pp. 2290-2296, 2014.

[11] S. Anitha, B. Brabu, D. J. Thiruvadigal, C. Gopalakrishnan, and T. S. Natarajan, "Optical, bactericidal and water repellent properties of electrospun nano-composite membranes of cellulose acetate and ZnO," Carbohydrate Polymers, vol. 87, no. 2, pp. 1065-1072, 2012.

[12] Z. Zhang, C. Shao, Y. Sun et al., "Tubular nanocomposite catalysts based on size-controlled and highly dispersed silver nanoparticles assembled on electrospun silica nanotubes for catalytic reduction of 4-nitrophenol," Journal of Materials Chemistry, vol. 22, no. 4, pp. 1387-1395, 2012.

[13] V. V. T. Padil, N. H. A. Nguyen, A. Ševců, and M. Černík, "Fabrication, characterization, and antibacterial properties of electrospun membrane composed of gum karaya, polyvinyl alcohol, and silver nanoparticles," Journal of Nanomaterials, vol. 2015, Article ID 750726, 10 pages, 2015.

[14] J. Yang, Y. Liu, S. Liu, L. Li, C. Zhang, and T. Liu, "Conducting polymer composites: material synthesis and applications in 
electrochemical capacitive energy storage," Mater. Chem. Front., vol. 1, no. 2, pp. 251-268, 2017.

[15] R. Shimizu, T. Kawakami, Y. Takashima, T. Tsuruoka, and K. Akamatsu, "In situ synthesis of $\mathrm{Cu} / \mathrm{Ni}$ alloy nanoparticles embedded in thin polymer layers," RSC Advances, vol. 6, no. 23, pp. 18895-18898, 2016.

[16] Q. Guo, R. Ghadiri, T. Weigel et al., "Comparison of in situ and ex situ methods for synthesis of two-photon polymerization polymer nanocomposites," Polymers, vol. 6, no. 7, pp. 20372050, 2014.

[17] O. Urbanek, F. Pierini, E. Choińska, P. Sajkiewicz, M. Bil, and W. Świeszkowski, "Effect of hydroxyapatite nanoparticles addition on structure properties of poly(L-lactide-co-glycolide) After gamma sterilization," Polymer Composites, 2016.

[18] M. K. Corbierre, N. S. Cameron, M. Sutton et al., "Polymerstabilized gold nanoparticles and their incorporation into polymer matrices," Journal of the American Chemical Society, vol. 123, no. 42, pp. 10411-10412, 2001.

[19] C. N. R. Rao, G. U. Kulkarni, P. J. Thomas, and P. P. Edwards, "Metal nanoparticles and their assemblies," Chemical Society Reviews, vol. 29, no. 1, pp. 27-35, 2000.

[20] M. Brust, M. Walker, D. Bethell, D. J. Schiffrin, and R. Whyman, "Synthesis of thiol-derivatised gold nanoparticles in a twophase liquid-liquid system," Journal of the Chemical Society, Chemical Communications, no. 7, pp. 801-802, 1994.

[21] Y. Tauran, A. Brioude, A. W. Coleman, M. Rhimi, and B. Kim, "Molecular recognition by gold, silver and copper nanoparticles," World Journal of Biological Chemistry, vol. 4, no. 3, pp. 3563, 2013.

[22] A. Cirri, A. Silakov, L. Jensen, and B. J. Lear, "Chain length and solvent control over the electronic properties of alkanethiolateprotected gold nanoparticles at the molecule-to-metal transition," Journal of the American Chemical Society, vol. 138, no. 49, pp. 15987-15993, 2016.

[23] S. Ristig, O. Prymak, K. Loza et al., "Nanostructure of wetchemically prepared, polymer-stabilized silver-gold nanoalloys ( $6 \mathrm{~nm}$ ) over the entire composition range," Journal of Materials Chemistry B, vol. 3, no. 23, pp. 4654-4662, 2015.

[24] K. L. Kelly, E. Coronado, L. L. Zhao, and G. C. Schatz, "The optical properties of metal nanoparticles: the influence of size, shape, and dielectric environment," The Journal of Physical Chemistry B, vol. 107, no. 3, pp. 668-677, 2003.

[25] M. P. Mallin and C. J. Murphy, "Solution-phase synthesis of sub$10 \mathrm{~nm}$ Au-Ag alloy nanoparticles," Nano Letters, vol. 2, no. 11, pp. 1235-1237, 2002.

[26] Z. G. Hu, P. Prunici, P. Patzner, and P. Hess, "Infrared spectroscopic ellipsometry of n-alkylthiol (C5-C 18) self-assembled monolayers on gold," Journal of Physical Chemistry B, vol. 110, no. 30, pp. 14824-14831, 2006.

[27] M. Brust, J. Fink, D. Bethell, D. J. Schiffrin, and C. Kiely, "Synthesis and reactions of functionalised gold nanoparticles," Journal of the Chemical Society, Chemical Communications, no. 16, pp. 1655-1656, 1995.

[28] N. Sandhyarani and T. Pradeep, "Crystalline solids of alloy clusters," Chemistry of Materials, vol. 12, no. 6, pp. 1755-1761, 2000.

[29] E. Mansfield, K. M. Tyner, C. M. Poling, and J. L. Blacklock, "Determination of nanoparticle surface coatings and nanoparticle purity using microscale thermogravimetric analysis," Analytical Chemistry, vol. 86, no. 3, pp. 1478-1484, 2014.
[30] W. Shi, Y. Sahoo, and M. T. Swihart, "Gold nanoparticles surface-terminated with bifunctional ligands," Colloids and Surfaces A: Physicochemical and Engineering Aspects, vol. 246, no. 1-3, pp. 109-113, 2004.

[31] A. Gupta, S. Mandal, M. Katiyar, and Y. N. Mohapatra, "Film processing characteristics of nano gold suitable for conductive application on flexible substrates," Thin Solid Films, vol. 520, no. 17, pp. 5664-5670, 2012.

[32] A. Laforgue and L. Robitaille, "Fabrication of poly-3-hexylthiophene/polyethylene oxide nanofibers using electrospinning," Synthetic Metals, vol. 158, no. 14, pp. 577-584, 2008.

[33] J. Du, S. Shintay, and X. Zhang, "Diameter control of electrospun polyacrylonitrile/iron acetylacetonate ultrafine nanofibers," Journal of Polymer Science, Part B: Polymer Physics, vol. 46, no. 15, pp. 1611-1618, 2008.

[34] S.-H. Tan, R. Inai, M. Kotaki, and S. Ramakrishna, "Systematic parameter study for ultra-fine fiber fabrication via electrospinning process," Polymer, vol. 46, no. 16, pp. 6128-6134, 2005.

[35] J. R. Cardenas, M. G. O. de França, E. A. de Vasconcelos, W. M. de Azevedo, and E. F. da Silva Jr., "Growth of sub-micron fibres of pure polyaniline using the electrospinning technique," Journal of Physics D: Applied Physics, vol. 40, pp. 1068-1071, 2007.

[36] M. S. Enayati, T. Behzad, P. Sajkiewicz et al., "Fabrication and characterization of electrospun bionanocomposites of poly (vinyl alcohol)/nanohydroxyapatite/cellulose nanofibers," International Journal of Polymeric Materials and Polymeric Biomaterials, vol. 65, no. 13, pp. 660-674, 2016.

[37] A. Wurm, D. Lellinger, A. A. Minakov et al., "Crystallization of poly( $\varepsilon$-caprolactone)/mwcnt composites: a combined saxs/waxs, electrical and thermal conductivity study," Polymer (United Kingdom), vol. 55, no. 9, pp. 2220-2232, 2014.

[38] U. Stachewicz, R. J. Bailey, W. Wang, and A. H. Barber, "Size dependent mechanical properties of electrospun polymer fibers from a composite structure," Polymer (United Kingdom), vol. 53, no. 22, pp. 5132-5137, 2012. 

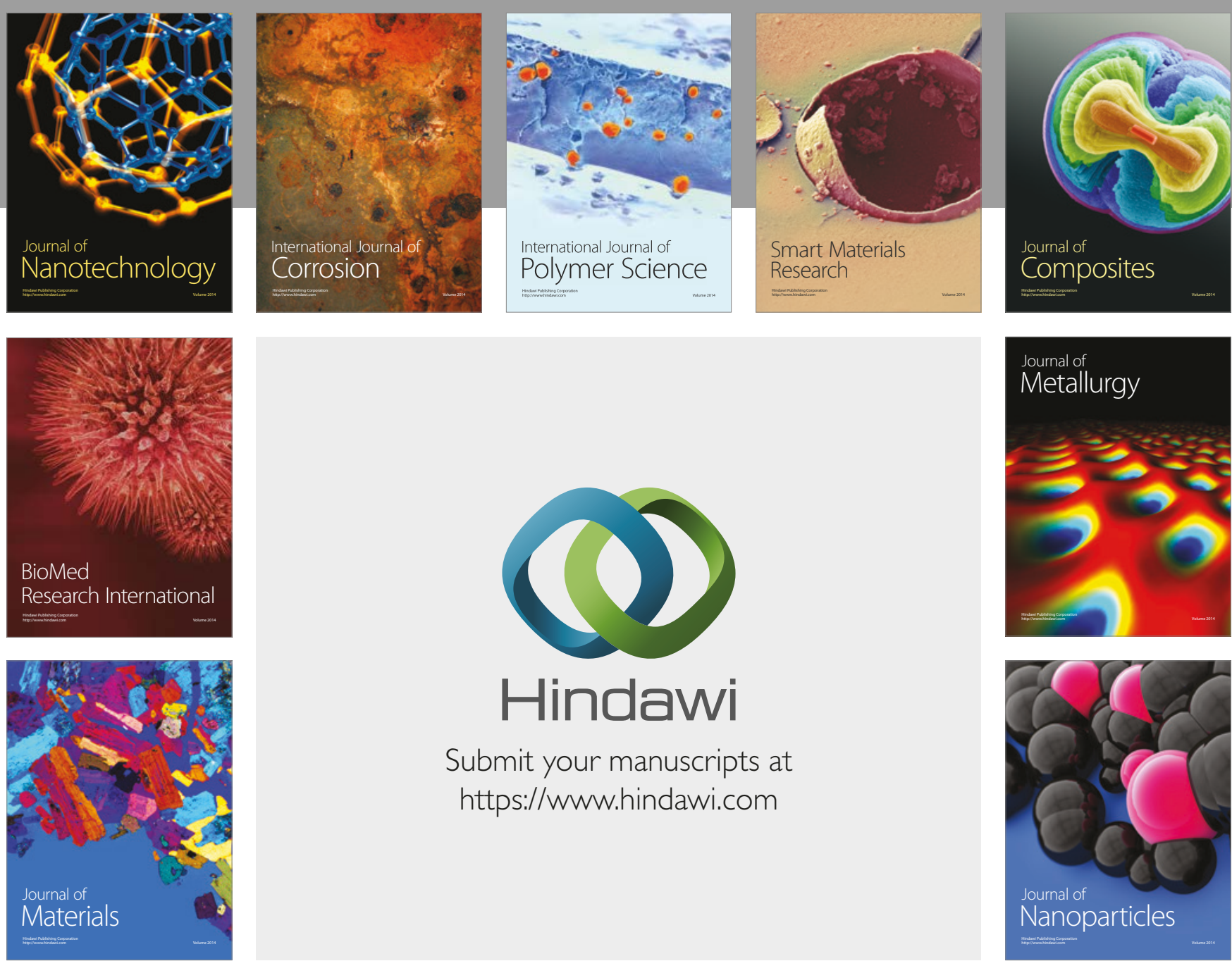

\section{Hindawi}

Submit your manuscripts at

https://www.hindawi.com
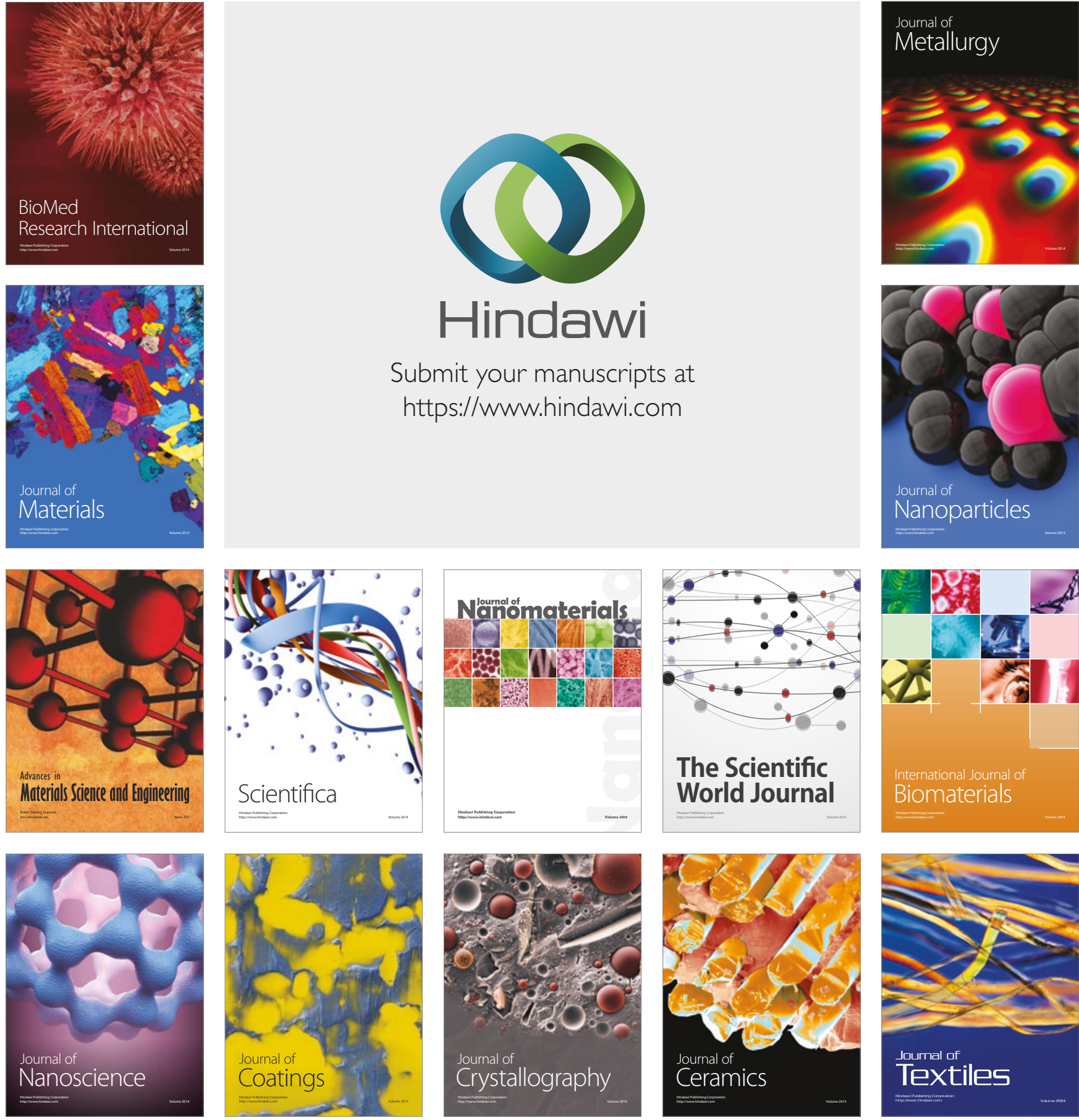

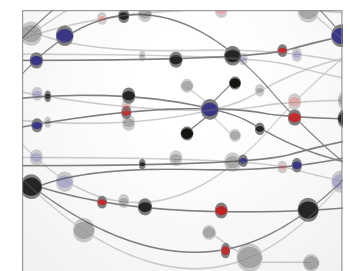

The Scientific World Journal
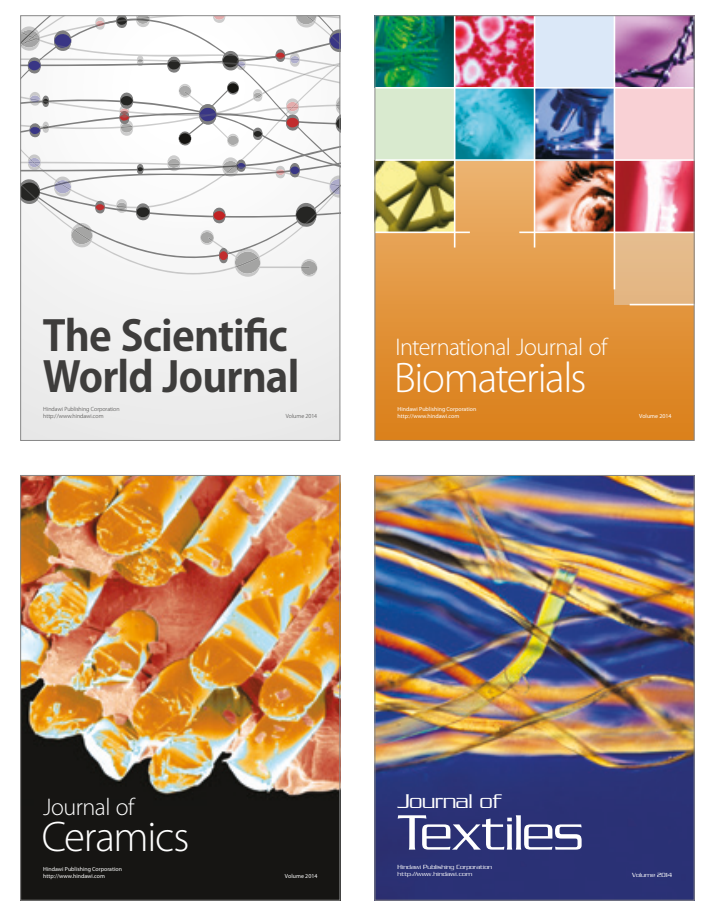